\title{
E-Learning im Vormarsch
}

Derzeit befindet sich das Hochschulsystem weltweit in einem tiefgreifenden Veränderungsprozess. Die Universitäten müssen sowohl mit den Bedürfnissen, Wünschen und Anforderungen der Studierenden als auch mit aktuellen Maßnahmen im Bereich der Hochschulpolitik schritthalten. Daher werden zunehmend Informationstechnologien und E-Learning-Systeme als wesentliche Faktoren bei der zukünftigen Ausübung der Lehrtätigkeit von Universitäten angesehen, sodass diese Institutionen mehr und mehr in Online-Systeme und -Geräte investieren. Im Zeitalter der Digitalisierung bleibt allerdings eine der Hauptherausforderungen der Universitäten die Integration und bestmögliche Nutzung von innovativen E-Learning-Systemen, um sowohl das Lehren als auch das Lernen zu optimieren und zu modernisieren sowie neue Lernerfahrungen aufzubauen und zu gestalten.

Trotz einiger Schattenseiten hat E-Learning zweifelsohne viele Vorteile. Anders als das Lernen von Angesicht zu Angesicht gewinnt E-Learning zunehmend an Popularität, vor allem wegen seiner Flexibilität bei der Vermittlung von Bildung und des barrierefreien Zugangs zu einem breiten Spektrum an Inhalten und Ressourcen. Daher hat E-Learning eine große Bedeutung für den Lernprozess, denn es hat das Potenzial, die Lernqualität zu verbessern, und bietet die Möglichkeit, Kurse zu personalisieren und an die Bedürfnisse der Lernenden anzupassen. E-Learaning erleichtert die Zusammenarbeit und erlaubt den Studierenden, in ihrem eigenen Rhythmus zu lernen. Darüber hinaus motiviert es sie, mit ihren Kommilitonen zu interagieren, zu diskutieren und Standpunkte sowie Ideen auszutauschen.

Die digitalen Lernkurse mit interaktiven Live-Segmenten und auch Augmented-Reality-Komponenten verbinden Theorie und Praxis, sodass auch Anwendungsbeispiele, Produkte und Prozesse, die in der Industrie zum Einsatz kommen, vorgestellt werden können. Das macht die Lerninhalte für die Studierenden lebendiger und interessanter und hilft, sie auf die Anwendung des neu erworbenen Wissens in ihrem zukünftigen Arbeitsfeld erfolgreich vorzubereiten. Es sind diese spannenden und anwendungsorientierten Einblicke, die E-Learning für die Studierenden so einzigartig machen.

Ihre ZWF-Herausgeber und-Redaktion

MES-System mit Fertigungskommunikation

Im Rahmen von Digitalisierung und Industrie 4.0 spielen Maschinen- und Bauteilkommunikation ebenso wie die fertigungsnahe Intralogistik eine große Rolle. Die FAUSER AG arbeitet daran, solche Möglichkeiten in ihre eng verzahnten ERP-. MES- und BDE-Module zu integrieren.

So entstehen in einem vom Bundesministerium für Wirtschaft und Energie (BMWi) geförderten Forschungsprojekt mit der ATS Elektronik GmbH, dem Institut für Fertigungstechnik und Werk- zeugmaschinen (IFW) der Leibniz-Universität Hannover und der FAUSER AG Funktionen für das Manufacturing Execution System der Zukunft. Das Titelbild zeigt die digitale Plantafel des MES-Core Modules.

\section{FAUSER AG}

Talhofstr. 30, 82205 Gilching

Telefon: +49 (81 05) 77 98-0

Telefax: +49 (81 05) 77 98-150

marketing@fauser.ag

www.fauser.ag

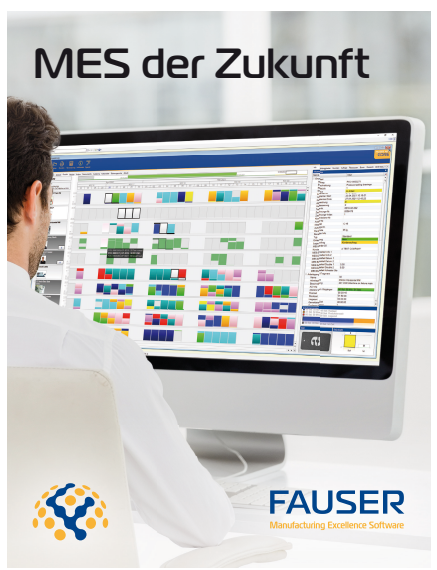

